\title{
"Cat Scratch Colon" in a Patient with Ischemic Colitis
}

\author{
Eui Ju Park, Joon Seong Lee, Tae Hee Lee, Dae Han Choi, Eui Bae Kim, Seong Ran Jeon, Su Jin Hong and \\ Jin-Oh Kim
}

Institute for Digestive Research and Digestive Disease Center, Department of Internal Medicine, Soonchunhyang University College of Medicine, Seoul, Korea

\begin{abstract}
"Cat scratch colon" is a gross finding characterized by hemorrhagic mucosal scratches on colonoscopy. It is usually associated with a normal colon and is rarely associated with collagenous colitis. In a previous report, cat scratch colon was noted in the cecum and ascending colon, but has also been observed in the distal transverse colon. The patient in this study was also diagnosed with ischemic colitis that may have played a role in the development of cat scratch colon.
\end{abstract}

Key Words: Cat scratch colon; Colonoscopy; Barotrauma; Ischemia; Colitis

\section{INTRODUCTION}

Cat scratch colon is a rare colonoscopic finding first described by McDonnell et al. ${ }^{1}$ in 2007 and is defined as bright, erythematous linear breaks that arise spontaneously and resemble scratches made by a cat. Sometimes, bright red linear markings with extravasations of fresh blood are seen in the cecum and ascending colon. The etiology is not well understood. In previous studies, cat scratch colon was a rare condition that occurs spontaneously or is partially associated with collagenous colitis. ${ }^{1-3}$ A large volume of air entering the lumen of the colon during colonoscopy was believed to cause cat scratch colon. ${ }^{4}$ The cecum and ascending colon are the most common regions of occurrence of cat scratch colon. However, we here report a case of ischemic colitis so that we can broaden our experience of this endoscopic finding in the transverse colon.

\section{CASE REPORT}

A 65-year-old woman was referred for colonoscopy because

Received: March 9, 2014 Accepted: April 27, 2014

Correspondence: Joon Seong Lee

Institute for Digestive Research and Digestive Disease Center, Department of Internal Medicine, Soonchunhyang University College of Medicine, 59 Daesagwan-ro, Yongsan-gu, Seoul 140-743, Korea

Tel: +82-2-709-9202, Fax: +82-2-709-9696, E-mail: joonlee@hosp.sch.ac.kr

(c) This is an Open Access article distributed under the terms of the Creative Commons Attribution Non-Commercial License (http://creativecommons.org/ licenses/by-nc/3.0) which permits unrestricted non-commercial use, distribution, and reproduction in any medium, provided the original work is properly cited. of abdominal pain and hematochezia. She had a history of end-stage renal disease, hypertension, and diabetes mellitus. She underwent hemodialysis three times per week. The patient's hemodynamic parameters were stable. On physical examination, she was found to have right abdominal pain and tenderness. Laboratory tests revealed a white blood cell (WBC) count of $13,900 / \mathrm{mm}^{2}$, a hemoglobin level of $9.4 \mathrm{~g} / \mathrm{dL}$, and a platelet count of $228,000 / \mathrm{mm}^{2}$. Serum biochemistry showed creatinine level, $6.6 \mathrm{mg} / \mathrm{dL}$; albumin level, $3.0 \mathrm{~g} / \mathrm{dL}$; alanine aminotransferase level, $36 \mathrm{IU} / \mathrm{L}$; aspartate aminotransferase level, 38 IU/L; bilirubin level, $1.2 \mathrm{mg} / \mathrm{dL}$; and alkaline phosphatase level, 198 IU/L with normal electrolytes. Serum was negative for antinuclear antibody and HIV antibody. Anemia was associated with chronic renal failure. On stool examination, negative results were obtained for stool WBC, stool culture, and Clostridium difficile toxin A/B. The electrocardiogram showed normal results. An esophagogastroduodenoscopy revealed no evidence of bleeding. Colonoscopy was performed after standard bowel preparation and under sedation with 3 mg of midazolam. The colonoscopic findings showed diffuse bowel wall edema. Upon examination of the distal transverse colon, we found several deep linear red mucosal breaks resembling scratches (Fig. 1).

In the ascending colon, dark purple edematous mucosa and necrotic epithelial lesions were found (Fig. 2). Cecal intubation was not performed because of severe ischemic changes with necrosis. The remaining colorectal mucosa was grossly normal. Computed tomography (CT) scan showed moderate-to- 

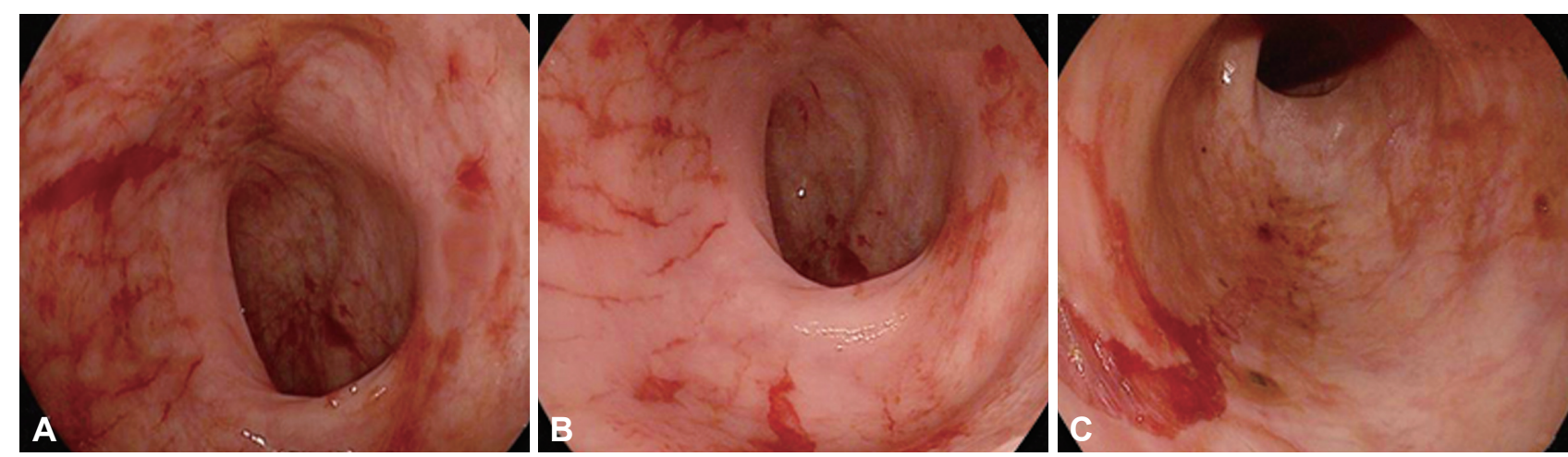

Fig. 1. (A-C) Photograph showing an endoscopic view of the middle-to-distal transverse colon. Bright red, linear, hemorrhagic lesions and scratch mucosal lesions are seen.
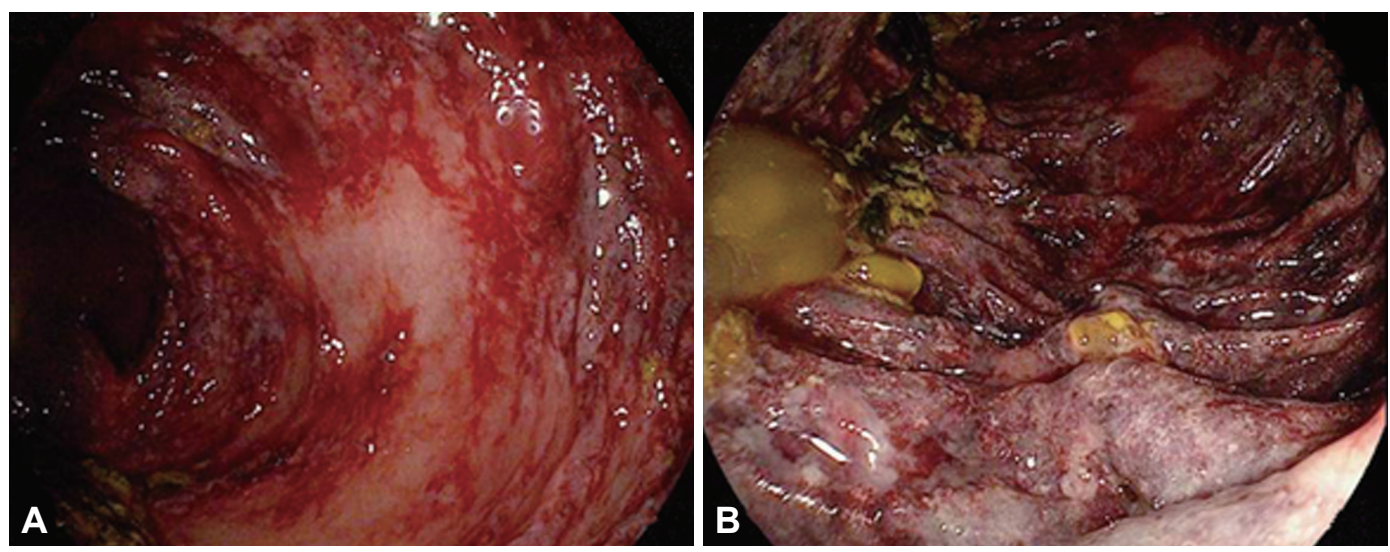

Fig. 2. Photograph showing an endoscopic view of the cecum (A) and ascending colon (B). Severe redness, swelling, and an almost a bluish appearance of the wall of the bowel are seen.

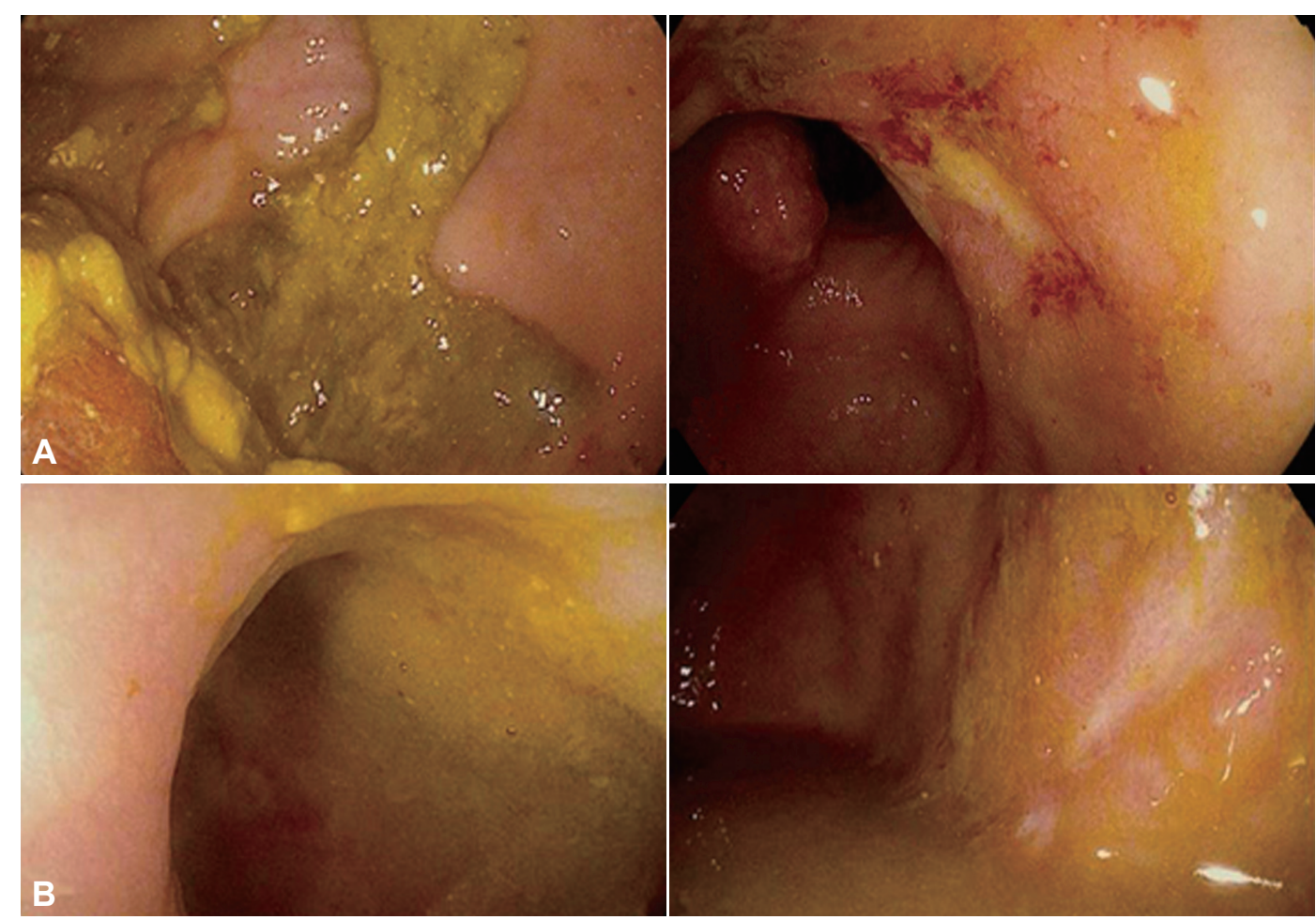

Fig. 3. After 3 weeks, a follow-up colonoscopy reveals mild wall edema, with erythematous and scarred mucosa of the cecum and ascending colon (A). Mucosal scarring is seen in the middle-to-distal transverse colon (B). 
severe diffuse bowel wall thickening and focally decreased mucosal enhancement in the right colon. The patient was diagnosed with ischemic colitis based on colonoscopic and CT findings, and clinical manifestations. The patient thus received supportive care. Intravenous fluids were administered to treat dehydration, and total parenteral nutrition with prophylactic antibiotics was provided. The patient was placed on bowel rest until the symptoms resolved. After 3 weeks, follow-up colonoscopy and CT were performed. On follow-up colonoscopy, the previous severe ischemic colonoscopic findings had disappeared, and ischemic mucosal change showed further resolution (Fig. 3). No further mucosal tearing was observed during the follow-up colonoscopy. On CT scans, improvement in ischemic colitis was noted. The patient no longer complained of abdominal pain or hematochezia.

\section{DISCUSSION}

"Cat scratch colon" was described for the first time in 2007. Over the past few years, very few cases have been reported. These lesions have a prevalence rate of $0.25 \% .{ }^{1}$ Cat scratch colon is defined as the presence of linear mucosal breaks in the cecum and ascending colon; the scratches are of variable length, bright red, resembling a cat scratch. Although the cause is unclear, there are several hypotheses: Barotrauma from air insufflation into a less compliant colon during colonoscopy, ${ }^{1}$ complications resulting from collagenous colitis, ${ }^{2,3}$ tension in the wall of a cylindrical vessel, ${ }^{5}$ lesions related to diversion colitis, ${ }^{4}$ chronic cholestasis and its complications, ${ }^{6}$ chronic anti-inflammatory drug ingestion, ${ }^{7}$ and others. Until recently, the main proposed causative mechanism has been barotrauma due to excess insufflation of air. Recently, colonic perforation due to high air pressure was reported following observation of a cat scratch colon. ${ }^{8}$ This supports the hypothesis that cat scratch colon is commonly associated with barotrauma etiology. However, barotrauma alone is not the sole cause of cat scratch colon. Recently, significant hemorrhagic linear marks have been reported to occur during colonoscopy in patients with collagenous colitis. ${ }^{1,2}$ Variable amounts of chronic inflammatory infiltration is typically present in the lamina propria, with an increased number of intraepithelial lymphocytes. The deposition of subepithelial collagen makes the bowel wall stiff and non-distensible. This lack of compliance may aggravate superficial mucosal injury from excessive insufflation during colonoscopy.

In the case documented above, cat scratch colon was seen in a patient with ischemic colitis. The right colon is predominantly involved in the hemodialysis population. ${ }^{9}$ Hemodialysis tends to cause repeated hypotensive episodes and may thus in- duce vasoconstriction of the vasa recta in the right colon, leading to colonic ischemia. ${ }^{10}$ Ischemic colitis is caused by hypoxemia to the terminal vascular distribution of the intestines. The ischemic process is transient and limited to the mucosa, with fibrosis and formation of colonic strictures in the late phase. Chronic ischemic change may play a role in cat scratch colon development owing to decreased compliance similar to collagen colitis. Cat scratch colon predominantly occurs in the right colon, where the mucosa is less compliant and more susceptible. ${ }^{8}$ Until recently, cat scratch colon was reported in only the cecum and ascending colon. Unlike other cases of cat scratch colon, this patient's lesion was located in the middle-to-distal transverse colon. In this case, the mucosa of the ascending colon and proximal transverse colon was already edematous, because of which barotrauma may have been an unlikely cause. Moreover, the ischemic parts of the colon wall were too stiff (decreased compliance), and pressure during air insufflation may have affected the more susceptible distal ischemic area. In our case, we did not perform a mucosal biopsy and could not exclude collagenous colitis entirely. In conclusion, the presence of cat scratch colon during colonoscopy may suggest barotrauma or other colonic pathology that affects wall compliance. Ischemic colitis in this patient may have played a role in the development of cat scratch colon.

\section{Conflicts of Interest}

The authors have no financial conflicts of interest.

\section{REFERENCES}

1. McDonnell WM, Loura F, Pointon MJ, Greenson JK. Cat scratch colon. Endoscopy 2007;39:459-461.

2. Cruz-Correa M, Milligan F, Giardiello FM, et al. Collagenous colitis with mucosal tears on endoscopic insufflation: a unique presentation. Gut 2002;51:600.

3. Fasoulas K, Terzoudis S, Lazaraki G, et al. Cat scratch colon: an endoscopic finding suggesting collagenous colitis. Ann Gastroenterol 2010;23: 311-313.

4. Baudet JS, Diaz-Bethencourt D, Arguiñarena X, Soler M, Morales S, Avilés J. Cat scratch colon is caused by barotrauma secondary to insufflation during colonoscopy. Endoscopy 2008;40:878.

5. Yarze JC. Finding mucosal tears in collagenous colitis during colonoscopic insufflation. Gut 2003;52:613-614.

6. Purnak T, Ozaslan E, Yildiz A, Efe C. The cat scratch colon sign in a patient with chronic cholestasis. Endoscopy 2010;42(Suppl 2):E117.

7. Payeras G, Briz R, Barranco R, Calvache A, Castro P. Cat scratch colon. A new ethiopathogenic possibility. Rev Esp Enferm Dig 2010;102:720-721.

8. Couto Wörner I, Arévalo Gómez A, Alonso Aguirre PA. Cat scratch colon. Clin Gastroenterol Hepatol 2012;10:A24.

9. Longo WE, Ballantyne GH, Gusberg RJ. Ischemic colitis: patterns and prognosis. Dis Colon Rectum 1992;35:726-730.

10. Flobert C, Cellier C, Berger A, et al. Right colonic involvement is associated with severe forms of ischemic colitis and occurs frequently in patients with chronic renal failure requiring hemodialysis. Am J Gastroenterol 2000;95:195-198. 